\title{
Brain Tumor Detection By Various Image Segmentation Techniques With Introducation To Non Negative Matrix Factorization
}

\author{
Sahil J Prajapati ${ }^{1}$, Kalpesh R Jadhav ${ }^{2}$ \\ PG Student, Electronics \& Communication Department, Parul Institute of Engineering And Technology (PIET), \\ Vadodara, Gujarat, India ${ }^{1}$
}

Assistant Professor, Electronics \& Communication Department, Parul Institute of Engineering And Technology (PIET),

Vadodara, Gujarat ${ }^{2}$

\begin{abstract}
Image segmentation is one of the fundamental approaches of digital image processing. During past few years, brain tumor segmentation in magnetic resonance imaging (MRI) has become a popular research area in the field of medical imaging system. MRI is used in radiology for analysing internal structures and makes easy to extract the required region. Thresholding is the simple approach to introduce to the morphological operations which are useful for the detection of the tumor but not all tumor can be specifically detected by this technique so region growing is another technique which provide seed point approach to the segmenter ROI region so the tumor is easily detected and also further used for the classification purpose. Nonnegative Matrix Factorization is one of the most promising technique to reduce the dimensionality of the data.NMF has been applied earlier to the image Processing methods such as Pattern analysis and Text mining and now in this paper it is mainly used as a uninterruptable decomposition approach for detection of tumor and to further classify into various types and also for feature extraction.NMF aims to find two non negative matrices whose product closely approximate the original matrix.NMF contains all matrices to contain only non negative elements and the NMF results shows no cancellations, linear super position only and considerable sparsity.
\end{abstract}

Keywords: Image Segmentation, Magnetic Resonance Imaging(MRI),Threshold Segmentation, Region Growing Segmentation, Bhattacharya Coefficient.

\section{INTRODUCTION}

\section{NON NEGATIVE MATRIX FACTORIZATION}

Non negative matrix factorization is one of the most promising technique to reduce the dimensionality of data in terms of image processing. It is the most suitable matrix decomposition technique for pattern analysis tasks and also it is and also it is widely applied for clustering and latent feature extraction. Several types of objective functions have been introduced to explain this concept, but talking about NMF in terms of Brain tumor classification when the tumor is detected to by NMF is is easily used for the classification.NMF is a collections of the non negative terms so,it tries to find the non negative terms with reduced rank of approximation for a non negative data matrix .it is also useful in calculating the matrix evaluation in terms of sparsesity and specificivity. Matrix factorization method treat the data as an $\mathrm{m} *$ n matrix in which every column represents a data sample this matrix is approximated by by a product of two $\mathrm{k}$ matrices ,as follows:

\section{$\mathrm{A}=\mathrm{W} . \mathrm{H}$}

Where $\mathrm{A}$ is a data matrix, $\mathrm{W}$ is the $\mathrm{m}^{*} \mathrm{k}$ matrix of basis vectors and $\mathrm{H}$ is the $\mathrm{k} * \mathrm{n}$ matrix,that gives the co-ordinates of the sample in the feature space.for linear combinations of feature is given by

$$
A_{i j=\sum_{i=0}^{n}} \quad W_{i} H_{i j}
$$

From the above equations we can conclude that NMF has been widely used in mathematical background for increasing the interpretability and matrix factorizations has motivated us towards to calculate the matrix factorization of the face images and the tumor images so it play a wide role in enhancement to improve upon the accuracy of the images.

\section{BRAIN TUMOR INTRODUCTION}

Medical image processing is the most challenging and highly wanted field. Brain tumor detection in magnetic resonance imaging(MRI) has become an emerging field of medical image processing. Segmentation of images is one of the most difficult tasks holds an impotant position in image processing which determine the quality of the of the final result .Image segmentation is the process of dividing an image into different regions the aim of this paper is to provide a review on automated tool for brain tumor segmentation using MRI scanned image datasets .detection and extraction of tumor from MRI scan images of the brain is done by MATLAB software. 


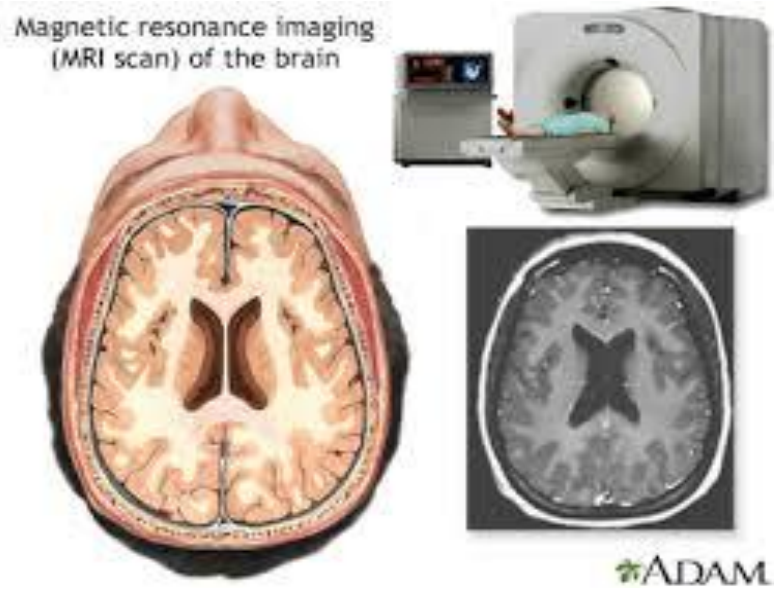

FIG-MRI scanner machine and image taken from the MRI scanner

A tumor is a thong of tissues that grows in an undisciplined manner that normalizes growth. Brain tumor is intrinsically serious and critical because of its persistent serious and infiltrative nature. Brain tumor is divided into two categories namely benign(non cancerous) and Malignent(cancerous) depending on the tumor beginning phase.

Benign brain tumors do not hold the malevolent cells, typically it can be removed and they hardly ever grew back. The margin of periphery of a benign brain tumor can be easily identified. These cells do not infect tissues around them or brodens to other parts of the body but still it can compress on resposive areas of the brain and cause severe health issues.

Malignant brain tumor contains cancerous cells and they are most likely to grow rapidly and invade in the surrounding healthly brain tissues. Very rarely cancerous cells may break away from from a malignent brain brain tumor and spread to the other parts of the body. The spread of cancer is called metastatis and it a type of secondary brain tumor which we are going to studied.

Imaging play a very important role in the diagonsis of brain tumors and recognizing a brain tumor generally involves a neurological assessment. Doctors use the diagonstic information to categorize from the least insistent to the most insistent identifying the tumor classes help the Doctors to verify the most suitable course of the treatment .one of the most preferable type of diagonstic method is MRI(Magnetic Resonance Imaging).MRI is a superior medical imaging technique used to generate high quality images of the human body with a high spatial resolution and tremendous discrimination of soft tissues. Anatomical information is used to observe human brain development and determine abnormalities.

There are many Segmentation techniques, based on similarity or discontinuity, such as threshold approach, Region growing approach. These approaches has now been improved to the Machine learning Approach.
Brain tumor classification include categorization of primary and secondary brain tumors into different classes .primary brain tumor that originate in the brain itself like Astrocytoma(AS), GliboblastromaMultiforme(GBM),Meni ngiomas(MEN), childtumorMedullostroma(MED),Seconda ry brain tumor are the cancer cells that originate from another part of the body and had spread to the brain like Metastatis(MET) tumors.

\section{BHATTACHARYA COEFFICIENT}

The probability error $P_{e}$ is an optimal measure of effectiveness of the set of features selected for the purpose of pattern recognition.owing to the difficulty involved in computation of probability error. The Bhattacharya Coefficient $(\rho-r h o)$, which was originally defined as the measure of overlap between two probability distribution ,has become a popular feature evaluation criterion in pattern recognition anf tumor classification. The two main reasons behind the popularity of $\rho$ in case of exponential family of distribution also used in Gaussian distribution or can say normal distribution.

Radiologists currently segment patients' tumors by hand on MR images before applying a treatment such as radiation therapy. This manual segmentation process is laborious and expensive. Automated tumor location could be used to seed or constrain an automated brain tumor segmentation system. the right part can be divided by an axis of symmetry. Tumors typically disturb this symmetry of the brain anatomy. We utilize this property to design a score, brain tumor locator score (BTLS), based on Bhattachaya coefficient (BC) as follows. In Fig.1 we divide the brain into two four quadrants: top-left, top-right, bottom-left and bottom-right. H's are normalized gray level histogram for the respective image quadrants. BTLS is defined as:

BTLS $(1)=B C(H)$

where, $\mathrm{T}$ stands for Top, $\mathrm{L}$ stands for left, $\mathrm{R}$ stands for right, D stands for down and $l$ stands for distance of the horizontal line from the horizontal axis and $B C(P, Q)$ is the Bhattacharya Coefficient between two normalized histograms $P$ and $Q$ : The value of BC lies between 0 and 1. It achieves a value of 1 when $P$ and $Q$ are equal. The more similar $P$ and $Q$ are the closer is the value of the Bhattacharya coefficient to unity. To reveal the nature of the score BTLS we plot it against the distance $l$ in Fig. 2. Note that there is a highest peak and there is a lowest valley in the BTLS plot these two points correspond respectively to the top and the bottom of the tumor. Once the top and the bottom bounding lines of the tumor is found, we repeat the procedure to find the left and the right bounding lines similarly to finally obtain the bounding box around the tumor.

The algorithm is real-time and suitable for indexing tumor images for archival purposes. In database applications the typical query will be based on the location of the tumor as well as the size of the tumor that can be approximately indicated by the bounding box created by the proposed 
algorithm. We compute three performance metrics to evaluate the proposed method- (a) detection score, (b) segmentation score and (c) location score as follows:

Detection score $=\mathrm{G} \cap \mathrm{S} \div \mathrm{S}$

\section{Segmentation score $=\mathrm{G} \cap \mathrm{S} \div \mathrm{GUS}$}

where $G$ is the set of the tumor pixels found by a radiologist and $S$ is set of pixels found by the proposed algorithm. Note that the detection score does not penalize the result as long as it is inside the bounding box found by the expert. Because a potential application is to seed a segmentation algorithm with the bounding box found by the proposed method, Detection Score can be used to realize the efficacy of this seeding. For the purpose of indexing we describe the segmentation score and the third score:

Location score=Distance between centroid of $\mathrm{G}$ and $\mathrm{S}$.

\section{RESULTS OF BHATTACHARYA COEFFICIENT}

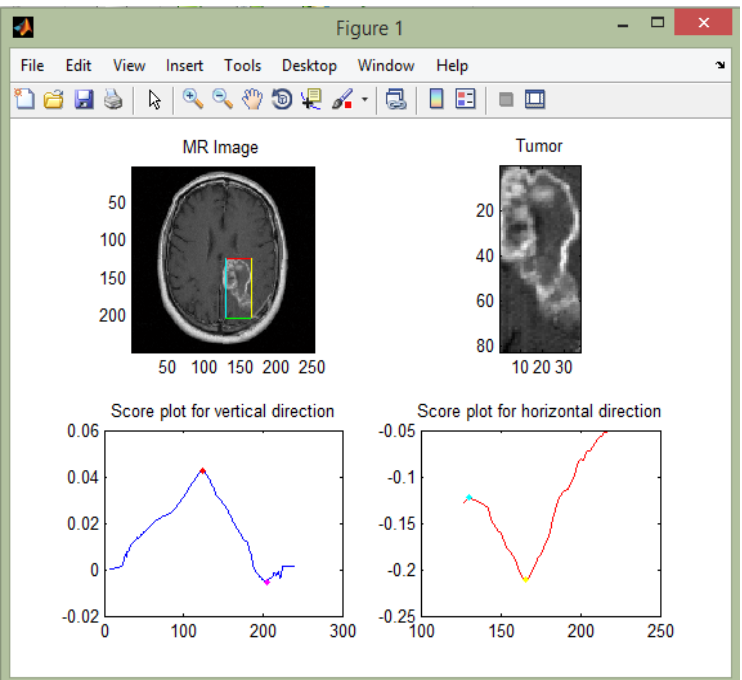

Fig-Brain tumor detection by bhattacharya coefficient on Child Tumor MRI image

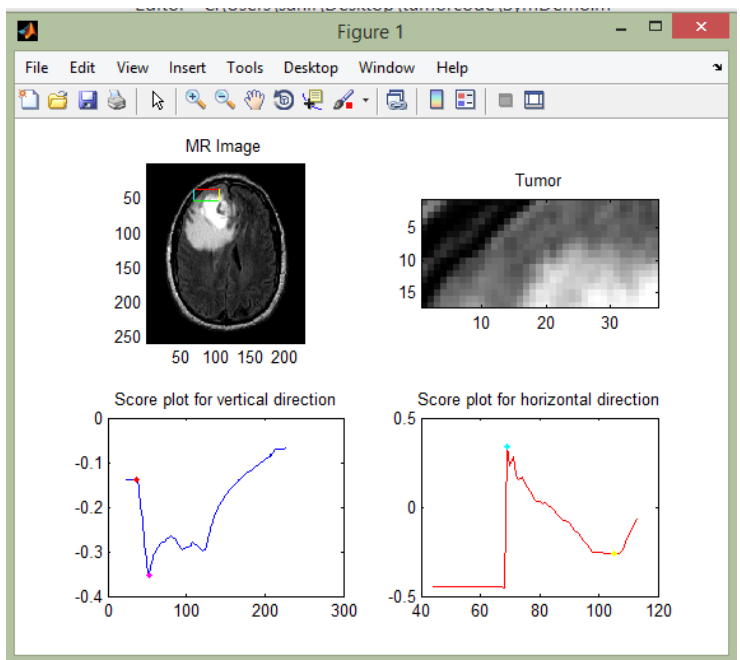

Fig-Brain tumor detection by bhattacharya coefficient on Cancer MRI image
II. METHODOLOGY

THRESHOLDING APPROACH IN DETECTION OF TUMOR

- Grayscale imaging

- Histogram equalization

- High pass filter

- Median filter

- Threshold segmentation

- Morphological operation

- Image Subtraction

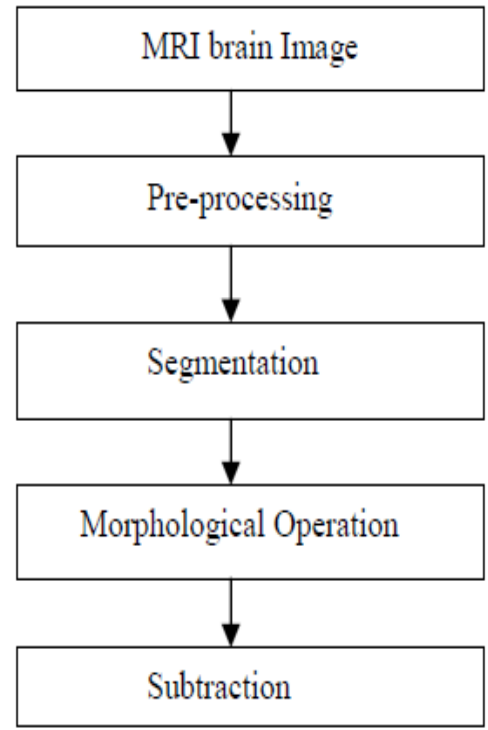

Fig-Architecture of Thresholding Approach

\section{Grayscale imaging}

Gray scale imaging is sometimes called "black and white",but technically this is misnomer in true black and white ,also known as halftone, and the only possible shades are pure black and pure white gray shading in a halftone image is obtained by considering the images as a grid of black dots on white background (or vice versa) and the sizes of the individuals dots determine the apparent lightness of the gray in their vicinity. The lightness of the gray is directly proportional to the number representing the brightness levels of the colors. Grayscale imaging can be collectively called as the as the ranges of shades of gray. Grayscale can be collectively called as the ranges of the shades of gray.MRI images are used in the preprocessed into a grayscale images

\section{HISTOGRAM EQUALIZATION}

Histogram equalization is a grayscale transformation that results in an image with a more or less flat histogram. In MATLAB software histogram of the images can be constructed by using imhist command.

\section{HIGH PASS FILTER}

High pass filter is used to do the sharpening of the images to the grayscale images. shapening is used to get the fine details of the image highlighted. Also it is used for edge detection. These filters sharpens images by creating a high contrast overlay that emphasis edge in the image ,so 
also we can say that enhanced image is the result of addition of original image and the scaled version of the line structure and edges in the image. High pass filter is also used to retain the frequency information within the image

\section{THRESHHOLD SEGMENTATION}

Segmentation is the process of partitioning the images into multiple segments.(set of pixels). Image segmentation is typically used to locate the objects and boundaries(lines, curves) in the images also we can say assining the label to each pixels in an image such that pixels share same label to view the visual characteristics. Threshold method is based on the threshold value to turn a grayscale image into a binary image.

\section{MORPHOLOGICAL OPERATION}

Morphology refers to the description of the properties of the shape and structure of the objects. Here binary images consists of various imperfections. Thresholding are distorted by the noise and texture featurs. Morphological operations are logical transformation based on the comparision of the pixel neighbourhood with a pattern. These operations are usually performed on the binary images where the pixels values is between 0 and 1 .

\section{IMAGE SUBTRACTION}

Image subtraction operators takes two images as input and produce as output a third image, whose pixels values are the values obtained by subtraction between the two image. in this technique the tumor is extracted based on the closely packed pixels present in the image.by this tumor is removed.

\section{RESULTS OF THRESHOLD APPROACH}

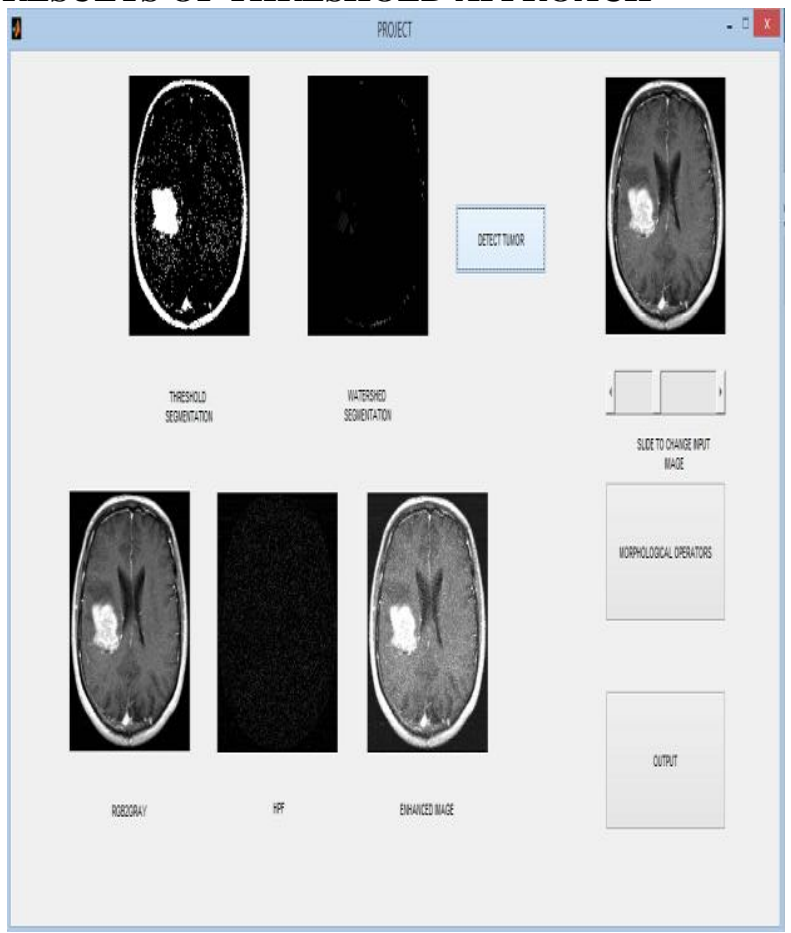

Fig-Brain tumor detection by thresholding approach with certain morphological operations.

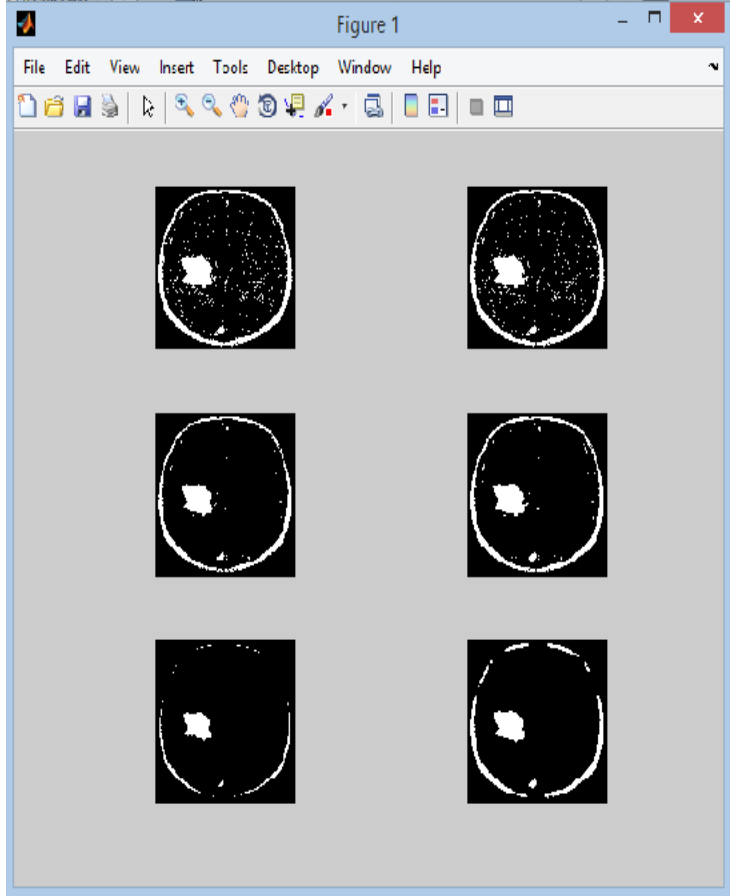

Fig-Thresholding basic operations are being performed on image-like Preprocessing, histogran equalized image, contrasting, edge detection, high pass image, with threshold segmentation along with image subtraction

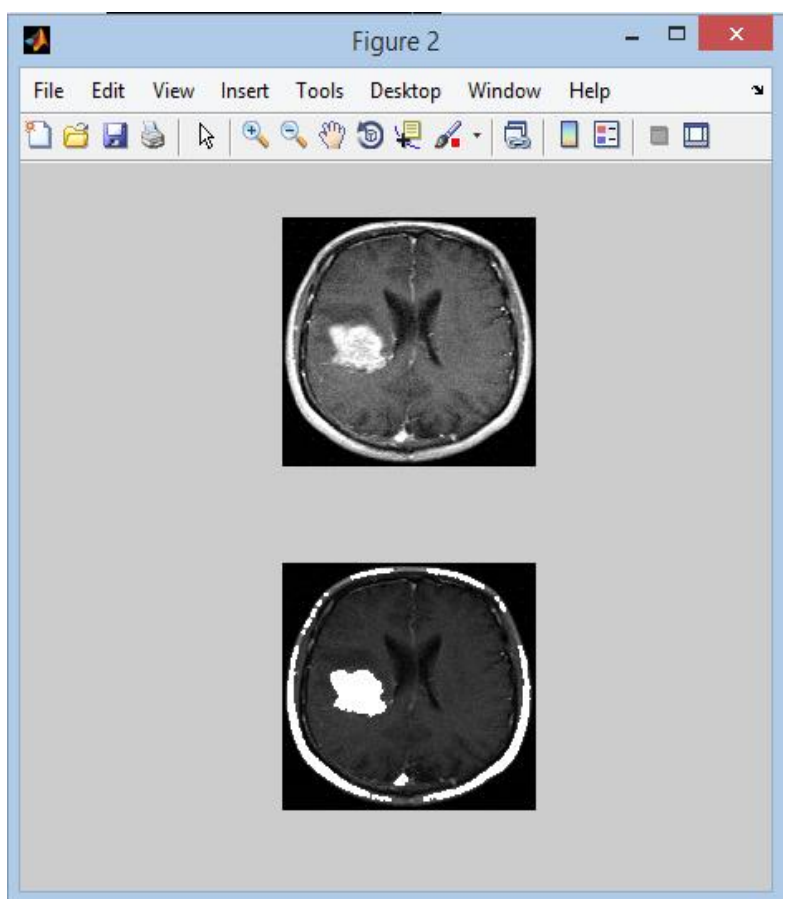

Fig-Image got after performing Morphological operationwith detection of the tumor region

\section{REGION GROWING APPROACH FOR} DETECTION OF TUMOR

Region Growing method is widely used for the Sementation of the ROI region of the tumor image then by the edge detection we can select the boundary points for finding the image regions. in region growing a seed point is the starting point for region growing and its selection 
could be done manually or automatically. In region growing the level set approach is used to achieve the exact edges of the tumor boundary which is useful in accurate estimation of its volume .In the region growing segmentation, the aim is to detemine the seed points. A seed point is the starting point for region growing and selection is vital for segmenting the results.

The principle is a user views are based on personal judgement, choose the seed point by the various approach like mouse based point and click mechanism. some MATLAB functions are employed in order to obtain the seed point. After ROI next step will be the edge detection operation for segmenting boundary.

\section{REGION GROWING ALGORITHM}

The first region growing method was the seeded region growing method. This method task was to take a set of seeds as input along with the image. The seed mark each of the object to be segmented the region are grown by comparing all unallocated neighbourhood pixels to the regions the difference between a pixel density value and the region mean $\delta$,is used as a measure of similarity the pixel with the smallest difference measred this way is allocated to the respective regions.the two main approaches are "Bottom-up" region growing and secondly "top bottom " region splitting and merging approach but we will go for first approach.

\section{ALGORITHM}

- Start from seed point.

- look at its neighbourhood.

- for each point $\mathrm{p}$ in nhood.

- $\quad$ if $P(p)=$ true then include pin region $\mathrm{L}$.

- else $\mathrm{p}$ is the border.

- in each iteration,look at the neighbour of each point in $\mathrm{L}$, to save computation ,"order" the point in the $\mathrm{L}$ and don,t check the points that have high score (e.g. the oncs that are in the middle of the region).

\section{RESULTS OF REGION GROWING APPROACH}

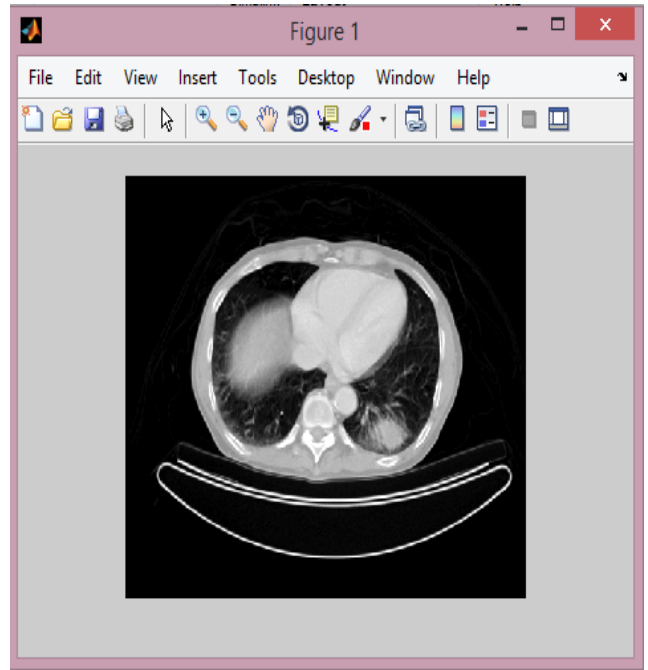

Fig-Original image of Brain with tumor

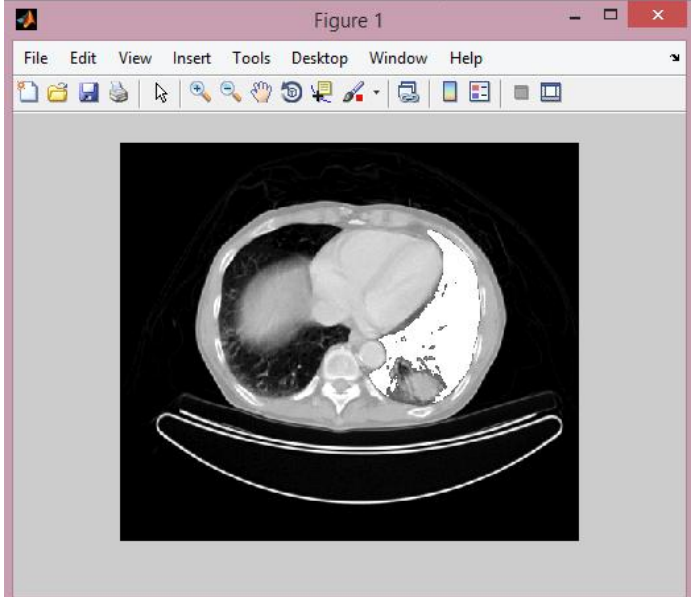

Fig-region grown image after performing certain operations.

\section{CONCLUSION}

We can conclude that morphological operations technique have proved to be very helpful in various image extraction and filtering techniques. The morphological operators can change the structuring elements of the image according to their use. Some operators llike open, erode, dilate, close ,boundingbox, regioncrop, have proved to be helpful in extractiong the brain tumor from brain MRI image. threshold segmentation was used to work on the desired region of the image. Thus applying image subtraction we can get the final brain tumor image. Also from studying the paper we can conclude that region growing technique is proved to be very promising in mostly the detection part in future we can classify the images of the brain tumor and classify which type of tumor is it of.NMF technique has also proved to be benefical in the classification of the tumor part so it use can be implemented accordinngly.

\section{REFRENCES}

[1] Natarajan P,Krishnan N,Natasha Sandeep Kenke,Shairya Nancy,Bhuvanesh Pratap Singh,"Tumor Detection Using threshold Operation In MRI Brain Images"IEEE international conference on computional intelligence and computing research,2012.

[2] Navneet Kaur,Mamta Juneja,"Brain Tumor Detection,Demarcation and Quantification via MRI",International Journal Of Computer Applications,IJCA vol87-no.18,February 2014.

[3] B.Jegatheeskumar,Dr.P.Subbaiah,Dr.P.Ramesh,"Various

Techniques for Brain Tumor Identification and segmentation Approach in MRI Images ",International Journal Of Computer Technology and Applications,IJCTA,Vol-5,Sep-Oct-2014.

[4] Nailah Afshan,Shaima Qureshi,Syed Mujitba Hussian,"Comparitive Study Of Tumor Detection Algorithms",2014 IEEE Conference On Medical Imaging and emerging Communication Algorithm,2014.

[5] Rajesh Patil,Dr. A S Bhalchandra,"Brain Tumor Extraction From MRI images using MATLAB",International Journal Of Electronics \& Communication Of Engineering And Soft Computing,IJECSSE,vol-2 Issue-1,2014.

[6] Jacek M Zurada,Tolga Ensari,Ehsaan Hosseini Asl,Jan Chorwski,"Non Negative Matrix Factorization And Its Applications To Pattern Analysis And Text Mining",2013 Federal Conference On Computer Science And Information System,IEEE 2013.

[7] S Ray,"On A Theoritical Property Of The Bhattacharya Coefficient As A Feature Evaulation Criterion",Pattern Recognition Letters,North Holland,1989.

[8] Aman chandra kaushik,Vandana Sharma,"Brain Tumor Segmentation From MRI Images And Volume Calculation Of Tumor",International Journal of Pharmaceutical Science Invention, Vol.2,Isuue-7,July-2013. 